\title{
Variations in the Volumes of Microbial Cells with Change in the Agitation Rates of Chemostat Cultures
}

\author{
By D. A. JOHN WASE* AND YATISH R. PATEL \\ Biochemical Engineering Section, Department of Chemical Engineering, University of \\ Birmingham, PO Box 363, Edgbaston, Birmingham B15 2TT, UK
}

(Received 2 November 1984)

\begin{abstract}
During continuous cultivation of Bacillus cereus, Staphylococcus epidermidis, Saccharomyces cerevisiae and two different strains of Escherichia coli, mean cell volume was found to be directly proportional to the agitation rate of the fermenter. For any one piece of equipment and microbial strain, highly reproducible linear relationships were measured, with similar intercepts and gradients for aerobic and anaerobic cultures independent of overall medium concentration, gradients for nitrogen-limited culture being always greater than those for carbon-limited culture. Alternative fermenter configurations or use of a different microbial strain furnished similar linear relationships, but slope and intercept were changed, indicating a dependence both on the geometry of the fermentation system and also on the microbial strain.
\end{abstract}

\section{INTRODUCTION}

Sizes of microbial cells have for many years been known to vary with cultural conditions. Thus, in batch culture, cells are largest just after the end of the lag phase (Dean \& Hinshelwood, 1966), whilst starvation following growth can cause dwarf forms as a possible prelude to film formation, often with exaggeration of the phenomenon at interfaces (Kjelleberg et al., 1983; Marshall, 1980). Excess of nutrient can cause the opposite effect, as in the continuous cultivation of yeast, when excess of glucose as non-limiting nutrient increases cell size, particularly at low dilution rates, due to accumulation of carbohydrate (Herbert, 1961). A change in nutrient limitation from carbon to nitrogen produces morphological changes in certain yeasts, leading to elongated forms, ascribed to changes in protein sulphydryl reductase levels (Brown \& Hough, 1965). Variations in the osmotic pressure of media can influence the sizes of microbial cells in both batch and continuous modes (e.g. Pirt, 1975) as can the imposition of a degree of synchrony on the culture (Dawson, 1972).

It is therefore clear that changes in the size of microbial cells may arise from any of a number of possible changes in the cell's environment. Sometimes the phenomenon is readily explained (e.g. Herbert, 1961), sometimes the explanation is more obscure (e.g. Brown \& Hough, 1965). However, none of the reports cited above exactly parallels or explains that described in the present paper. This present investigation was prompted by the discovery that continuously cultivated cells of Klebsiella pneumoniae varied in their resistance to ultrasonic disintegration (Nesaratnam et al., 1982). Further preliminary studies showed that one method of causing this change in resistance, even at constant dilution rate, was to alter the agitation rate (impeller speed). This finding suggested that the primary effect of the alteration in agitation rate was to change the mean cell volume in the culture. That this was so was confirmed when it was shown that the relationship between mean cell volume and agitation rate was indeed precisely linear (Wase et al., 1982). However, because these results were only for one micro-organism, over a limited range of agitation rates, a more detailed investigation was undertaken. This paper contains the results of this work. 


\section{METHODS}

Organisms, their maintenance and media for continuous cultivation. Escherichia coli strain B, a stock-culture from the Biochemical Engineering Section laboratory, was maintained on nutrient agar slopes stored at $4{ }^{\circ} \mathrm{C}$ and subcultured every month. Four growth media were used. Medium 1 was carbon limited and contained (per litre): glucose, $0.8 \mathrm{~g} ;\left(\mathrm{NH}_{4}\right)_{2} \mathrm{SO}_{4}, 0.5 \mathrm{~g} ; \mathrm{MgSO}_{4} .7 \mathrm{H}_{2} \mathrm{O}, 1.0 \mathrm{~g} ; \mathrm{KH}_{2} \mathrm{PO}_{4}, 4.0 \mathrm{~g}$; and $1 \mathrm{ml}$ of trace element solution which contained (per litre): $\mathrm{H}_{3} \mathrm{BO}_{3}, 100 \mathrm{mg} ; \mathrm{ZnSO}_{4} .7 \mathrm{H}_{2} \mathrm{O}, 40 \mathrm{mg} ;\left(\mathrm{NH}_{4}\right)_{6} \mathrm{Mo}_{7} \mathrm{O}_{24} .4 \mathrm{H}_{2} \mathrm{O}, 20 \mathrm{mg} ; \mathrm{CuSO}_{4} .5 \mathrm{H}_{2} \mathrm{O}$, $45 \mathrm{mg} ; \mathrm{FeSO}_{4} .7 \mathrm{H}_{2} \mathrm{O}, 250 \mathrm{mg}$ (Brown, 1966). Medium 2 was the same as medium 1 except that the concentrations of medium constituents other than trace elements were halved. Medium 3 was nitrogen limited. It contained (per litre): glucose, $3.0 \mathrm{~g} ;\left(\mathrm{NH}_{4}\right)_{2} \mathrm{SO}_{4}, 0.05 \mathrm{~g}$; and the rest of the components were the same as for medium 2 . Medium 4 was nitrogen limited. The chemicals used were the same as for medium 3 except that their concentrations were doubled. $E$. coli $\mathrm{NCIB} 10000$ was treated in exactly the same way as $E$. coli strain B.

Bacillus cereus BRL 2401 (Beecham Research Laboratories) was maintained on nutrient agar slopes stored at $4{ }^{\circ} \mathrm{C}$ and subcultured every month. Two media were used for growth. Medium 5 contained (per litre); glucose, $5.0 \mathrm{~g} ;\left(\mathrm{NH}_{4}\right)_{2} \mathrm{SO}_{4}, 0.5 \mathrm{~g} ; \mathrm{MgSO}_{4} .7 \mathrm{H}_{2} \mathrm{O}, 1.0 \mathrm{~g} ; \mathrm{KH}_{2} \mathrm{PO}_{4}, 4.0 \mathrm{~g}$; yeast extract, $2.0 \mathrm{~g}$. Medium 6 was the same except that the concentrations of all medium constituents were halved.

Staphylococcus epidermidis ATCC 9491 was maintained on nutrient agar slopes stored at $4{ }^{\circ} \mathrm{C}$ and subcultured every month. The medium composition (medium 7) was similar to that devised by Wimpenny \& Firth (1972) and contained (per litre): tryptone, $14 \mathrm{~g} ; \mathrm{KH}_{2} \mathrm{PO}_{4}, 0.70 \mathrm{~g}$; and $5 \mathrm{ml}$ of a salt solution containing (per litre): $\mathrm{MgSO}_{4} .7 \mathrm{H}_{2} \mathrm{O}, 10 \mathrm{~g} ; \mathrm{MnCl}_{2} .4 \mathrm{H}_{2} \mathrm{O}, 1 \cdot 0 \mathrm{~g} ; \mathrm{FeSO}_{4} .7 \mathrm{H}_{2} \mathrm{O}, 0 \cdot 4 \mathrm{~g} ; \mathrm{CaCl}_{2} .6 \mathrm{H}_{2} \mathrm{O}, 0 \cdot 1 \mathrm{~g}$. Glucose was sterilized as a separate solution and added aseptically to the bulk of the medium after sterilization, to $0.4 \%(\mathrm{w} / \mathrm{v})$. Antifoam (polypropylene glycol) $\left(1 \mathrm{mll}^{-1}\right)$ was also necessary to prevent foaming in the fermenter.

Saccharomyces cerevisiae NCYC 712 was maintained on slopes of MYGP agar [the malt extract/yeast extract/glucose/peptone medium described by Wickerham (1951) solidified with $2 \%(\mathrm{w} / \mathrm{v})$ agar], stored at $4{ }^{\circ} \mathrm{C}$ and subcultured every month. The growth medium (medium 8 ) was a modification of the medium described by Brown (1966). It was carbon limited and contained (per litre): glucose, $5.0 \mathrm{~g} ; \mathrm{KH}_{2} \mathrm{PO}_{4}, 4.0 \mathrm{~g} ; \mathrm{MgSO}_{4} .7 \mathrm{H}_{2} \mathrm{O}, 1.0 \mathrm{~g}$; $\left(\mathrm{NH}_{4}\right)_{2} \mathrm{SO}_{4}, 0.5 \mathrm{~g} ; \mathrm{CaCl}_{2} .6 \mathrm{H}_{2} \mathrm{O}, 0.25 \mathrm{~g}$; yeast extract, $0.1 \mathrm{~g}$. The medium was supplemented with $1 \mathrm{ml}$ of trace element solution (the same as that used for cultivation of $E$. coli). The $\mathrm{pH}$ was adjusted to $5 \cdot 0$ by addition of conc. $\mathrm{HCl}$ before sterilization.

Continuous culture apparatus. Three types of apparatus were used. The first was a baffled 0.5 litre New Brunswick 'Bioflo' continuous system (New Brunswick Scientific) incorporating agitation by means of a fourblade magnetically coupled impeller, aeration measurement and temperature measurement and control. $\mathrm{pH}$ was measured and controlled according to the experimental requirements. Dissolved oxygen tension was monitored by a galvanic probe linked to a New Brunswick model 40 dissolved oxygen meter. For continuous operation, spent medium and cells overflowed through a side arm.

A second continuous cultivation system, referred to as the 1.3 litre fermenter, was specially built in the departmental workshop. The fermenter consisted of an open-ended cylindrical Pyrex glass vessel of approximately 1 litre working volume, closed by EN 58J stainless steel end plates. Agitation to the baffled vessel was by means of a four-bladed glass-fibre reinforced polypropylene impeller, magnetically coupled to a shuntwound motor and controller. Aeration could be varied, dissolved oxygen measured, and $\mathrm{pH}$ and temperature controlled. For continuous operation, spent medium and cells overflowed through an adjustable weir tube, sealed through the base-plate by a compression fitting which clamped an O-ring.

The third fermenter used was a Bioengineering 'Baby' fermenter (Bioengineering UK, Caterham, Surrey). This was again of approximately 1 litre working volume, and consisted of a cylindrical glass vessel closed by stainless steel end plates. Temperature and $\mathrm{pH}$ were controlled. A mechanically sealed six-bladed demountable impeller assembly provided agitation; impellers of $4 \mathrm{~cm}$ diameter and of $6 \mathrm{~cm}$ diameter were used. The whole vessel and drive motor were mounted on a load cell, which provided a signal by which an associated microprocessor control unit operated an outflow pump set at a slightly higher rate than the medium inlet pump to permit continuous operation.

Continuous culture conditions. The essence of the experimental programme was that agitation rate should be varied as the experiment demanded, all other factors being kept constant. A dilution rate of $0 \cdot 1 \mathrm{~h}^{-1}$ and, for aerobic cultures, a volumetric air flow rate of 1 vol. vol. ${ }^{-1} \mathrm{~min}^{-1}$ were selected. However, for the first two fermenters described above, the working volume (and hence the dilution rate, which is the ratio of flow rate to working volume) varied with agitation rate. This variation arose either from general turbulence, which tended to throw more liquid through the side arm or over the constant-level weir, or from variations in gas hold up, or both. Preliminary experiments over a range of pumping rates and air-flow rates for each agitation rate required were therefore necessary to ascertain the exact pumping rate/volume combination furnishing a dilution rate of precisely $0.1 \mathrm{~h}^{-1}$ and an air-flow rate of $1 \mathrm{vol}$. vol. ${ }^{-1} \mathrm{~min}^{-1}$. These problems did not apply to the 'Baby' fermenter, where the contents were continuously weighed by the load cell.

For anaerobic cultures, care was taken to replace all silicone rubber medium inlet tubes by Tygon (which is far 
less permeable to oxygen), except for the short section in the peristaltic pump. A very slow bleed of nitrogen gas (white spot) was bubbled through the medium reservoir and also through the fermenter to ensure anaerobic conditions $\left(11 \mathrm{ml} \mathrm{min}^{-1}\right)$.

Control of $\mathrm{pH}$ was in all cases achieved by addition of sterile $0 \cdot 25 \mathrm{M}-\mathrm{NaOH}$.

Experience showed that, following a change in agitation rate, a minimum of three volume changes was necessary to achieve reproducible results, and normally at least five volume changes were allowed before samples were taken.

A summary of the cultivation conditions for individual organisms and experiments is given in Table 1, which also includes lines of best fit for the results, calculated by linear regression.

Analysis of steady-state cultures. Samples removed from the culture vessel were routinely checked for optical density at $650 \mathrm{~nm}$. Dry weight was obtained by centrifuging a known volume of culture, washing once at the centrifuge with deionized water, transferring the final sediment to a pre-weighed bottle, and drying to constant weight at $80^{\circ} \mathrm{C}$. Viable count was determined by conventional plating methods: samples were diluted and triplicate counts of each dilution were averaged $(E$. coli, B. cereus, Staph. epidermidis - nutrient agar; Sacch. ceretisiae - malt extract agar). Total cell counts and size distribution measurements were made by means of a Coulter Counter model $Z_{\mathrm{B}}$ (Coulter Electronics Co., Harpenden, Herts., UK), fitted with a $30 \mu \mathrm{m}$ orifice tube for E. coli, B. cereus and Staph. epidermidis and a $50 \mu \mathrm{m}$ orifice tube for Sacch. cerevisiae. Photographs were obtained with an Olympus model NH-2PC microscope equipped with a Polaroid-type camera (Olympus Optical Co.; obtained from Gallenkamp). To avoid Brownian movement, cell suspensions were separated from medium by filtration on $0.45 \mu \mathrm{m}$ Millipore filters. The suspensions were washed on the filter with $5 \%(\mathrm{w} / \mathrm{v})$ formalin in $1 \mathrm{M}$ saline, and then mounted in Vaseline petroleum jelly.

\section{RESULTS AND DISCUSSION}

\section{Correlation between agitation rate and mean cell volume}

Results of experiments involving continuous cultivation of $E$. coli strain B on a variety of media (experiments 1-9), are summarized in Fig. 1(a-d). In Fig. $1(a)$ (aerobic carbon-limited culture in the 1.3 litre fermenter), a precise linear correlation between agitation rate and mean cell volume is evident, analogous to the results previously reported for $K$. pneumoniae (Wase et al., 1982). The effect was considerable; increases in mean cell volume of the order of $50 \%$ were observed over the agitation rate range tested. Of the three experiments (1, 2 and 3$)$, two were duplicates ( 1 and 2), carried out sequentially. The resulting data demonstrate a high degree of reproducibility from experiment to experiment (as is confirmed by the lines of best fit and correlation coefficients listed in Table 1). The third set of data (obtained using half-strength medium) showed that whilst equilibrium cell numbers were diminished compared with the fullstrength medium (Table 2), this in no way altered either the absolute values of mean cell volume obtained for the various agitation rates tested, or the linear correlation between mean cell volume and agitation rate (Fig. $1 a$, Table 1), all points falling on the same straight line. Figure $1(b)$, anaerobic carbon-limited culture in the 1.3 litre fermenter, illustrates that as for the aerobic culture, there is a highly reproducible linear correlation between agitation rate and mean cell volume. Furthermore, statistical analysis of the data indicates that in all these cases, aerobic or anaerobic, the gradient and intercept are very similar (Table 1).

Results of analogous nitrogen-limited cultures (Fig. $1 c, d$ ) using the same apparatus were substantially similar, with one notable exception. Larger cells were always produced under nitrogen limitation than under carbon limitation for equivalent culture conditions. So the linear correlation between the mean cell volume and agitation rate was, again, very precise and highly reproducible, but whilst the intercept was similar to that found for both aerobic and anaerobic carbon-limited culture, the gradient was always steeper (Table 1, experiments 6-9). That nitrogen-limited culture always furnished larger cells than carbon limitation is not surprising, for carbon limitation is likely to restrict the availability of carbon-skeletal material: in contrast a change from aerobic to anaerobic culture changes the energy metabolism rather than the overall limitation, and therefore the mean cell volume is, as observed, not necessarily likely to change. However, neither of these considerations explained the changes in mean cell volume that occurred when agitation rate was varied.

Further results showed that the extent of these changes varied between strains. When $E$. coli NCIB 10000 was cultured anaerobically in the same apparatus (experiments 10 and 11 ; Fig. $2 a$, 


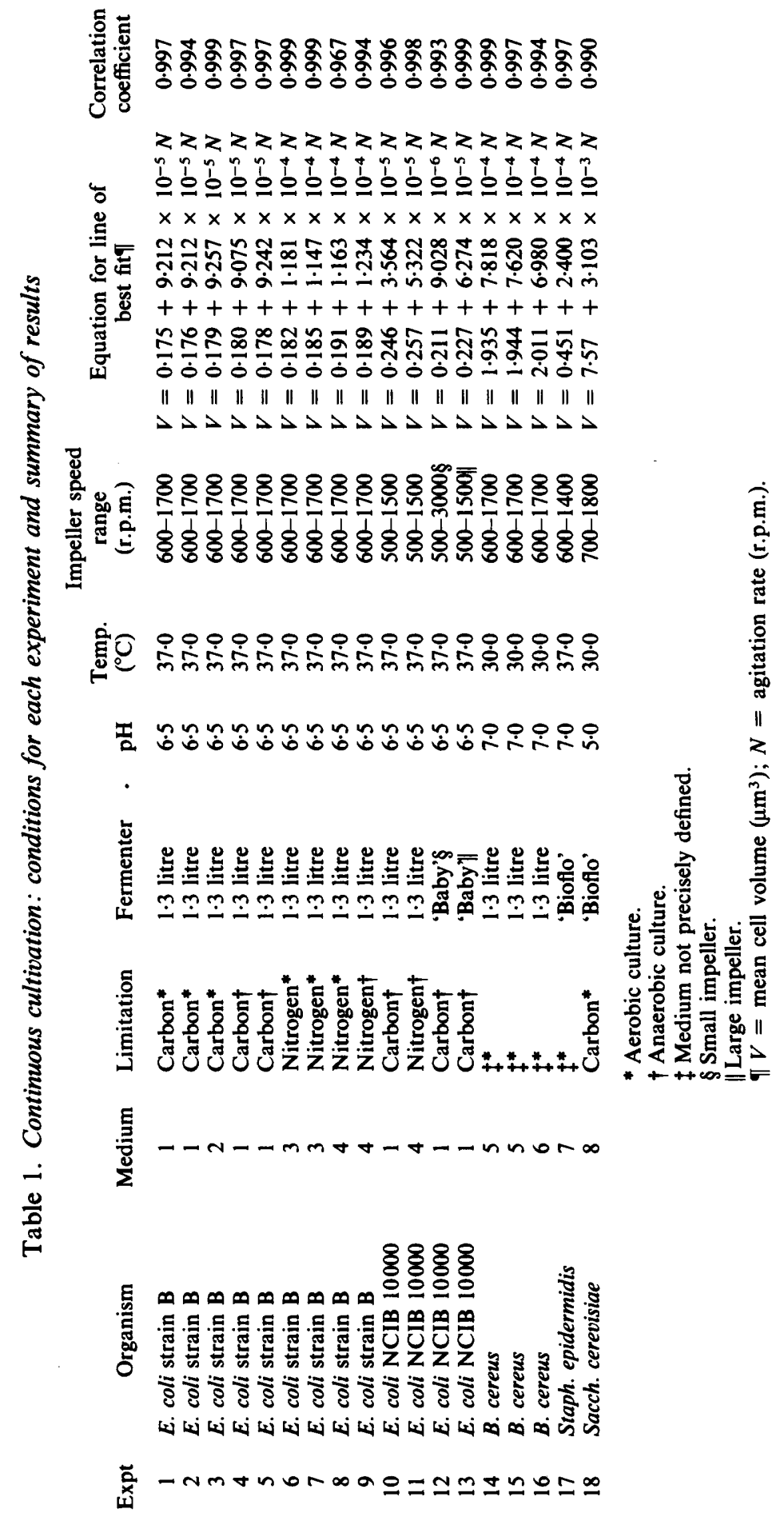




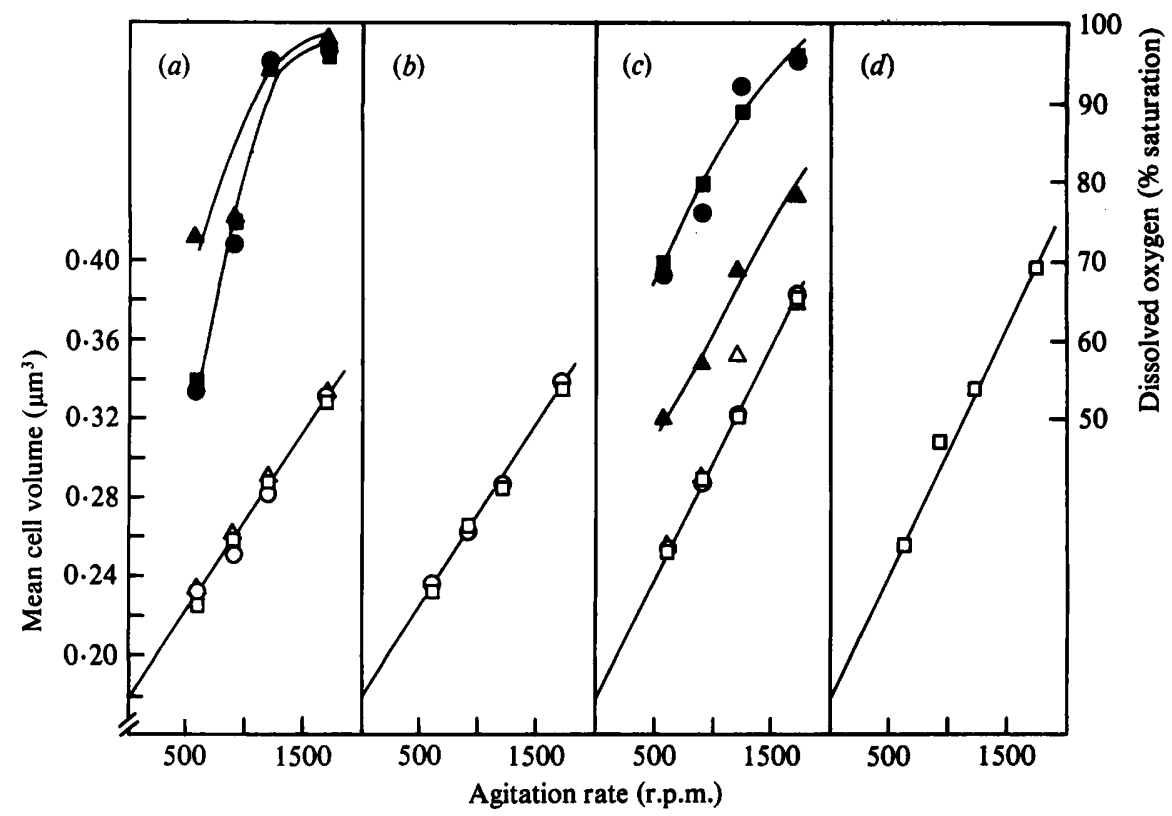

Fig. 1. Mean cell volumes of continuously cultivated $E$. coli strain B at various agitation rates. See Table 1 for details of the experiments. (a) Carbon-limited, aerobic. $\square, O, \Delta$, Mean cell volumes for experiments 1, 2 and 3; $\square, \bigcirc, \Delta$, dissolved oxygen for experiments 1,2 and 3. (b) Carbon-limited, anaerobic. $\square, O$, Mean cell volumes for experiments 4 and 5. (c) Nitrogen-limited, aerobic. $\square, O, \triangle$, Mean cell volumes for experiments 6,7 and $8 ; \square, 0, \Delta$, dissolved oxygen for experiments 6,7 and 8 . (d) Nitrogen-limited, anaerobic. $\square$, Mean cell volumes for experiment 9.

$b$ ), both the slopes and intercepts differed from those obtained for $E$. coli strain B, although overall, the effects were consistent with those previously obtained. Thus, whilst the slopes for $E$. coli NCIB 10000 were much less than those for $E$. coli strain B, nitrogen-limited cells were again larger than carbon-limited cells, and whilst the intercepts for $E$. coli NCIB 10000 were very much larger than those for strain B, they were, again, very similar to one another.

Because experiments in the 1.3 litre vessel were subject to certain potential errors in the continuous mode (see below), control cultures of $E$. coli NCIB 10000 were also carried out in the 'Baby' fermenter (experiments 12 and 13). Whilst the working volume of each vessel was of the same order, the arrangement of impeller, baffles, etc., differed and this is reflected in the results (Fig. $2 c, d$ : anaerobic carbon-limited culture). For each of the two impellers used in the 'Baby' fermenter, the relationship between agitation rate and mean cell volume was again precisely linear. However, the slopes and intercepts not only differed from those previously obtained with the 1.3 litre vessel, but also one from another. Thus for the larger impeller, slope and intercept were both larger than for the smaller impeller (Table 1). The extent of the effect we have observed therefore depends on the arrangement and geometry of the fermenter vessel.

The intercepts, obtained by extrapolation, were the theoretical mean volumes which would have been obtained at zero agitation rate. Whilst a non-agitated culture becomes subject to all sorts of constraints not necessarily associated with an adequately mixed system, it was of interest to see the practical results of such a determination. Anaerobic cultures were chosen to avoid oxygen mass-transfer problems: the culture was maintained anaerobic with a very slow bleed of nitrogen gas $\left(11 \mathrm{ml} \mathrm{min}^{-1}\right)$ and this was the only source of agitation. For $E$. coli strain B in the $1 \cdot 3$ litre fermenter under these conditions, mean cell volume was $0.186 \mu \mathrm{m}^{3}$ for carbon-limited and $0 \cdot 190 \mu \mathrm{m}^{3}$ for nitrogen-limited cells, which compares very favourably with the results in Table 1 . However, the significance of this 'constant' is currently not clear.

Organisms other than $E$. coli were also tested to see if the relationship between agitation rate and mean cell volume was of general application. Further results (from continuous cultivation of 


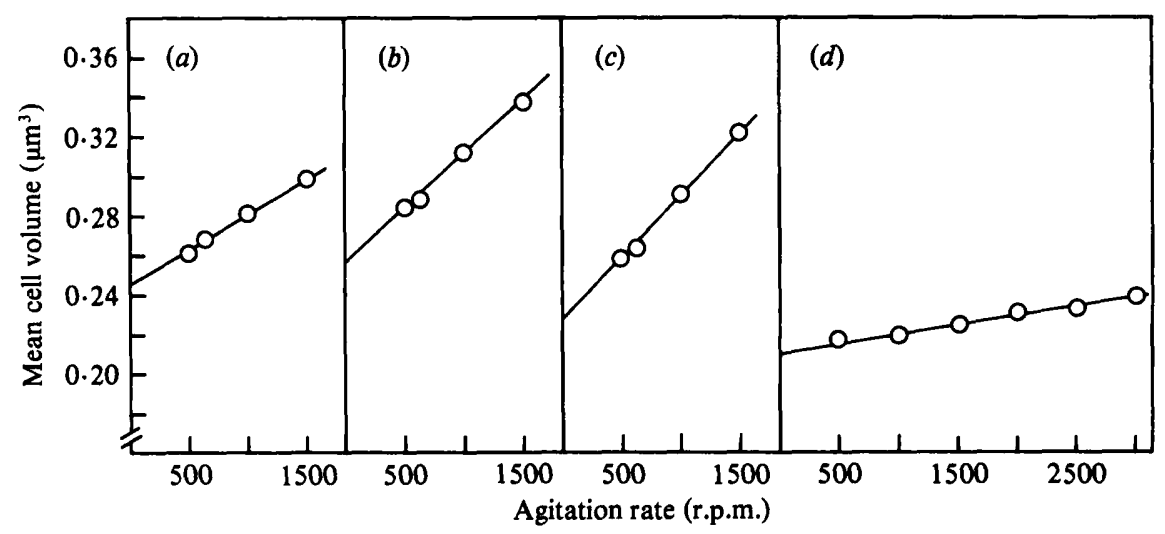

Fig. 2. Mean cell volumes of continuously cultivated E. coli NCIB 10000 at various agitation rates. All cultures were anaerobic. See Table 1 for details. (a) Experiment 10, carbon-limited, 1.3 litre fermenter. (b) Experiment 11, nitrogen-limited, 1.3 litre fermenter: (c) Experiment 13, carbon-limited, 'Baby' fermenter, large impeller. $(d)$ Experiment 12, carbon-limited, 'Baby' fermenter, small impeller.
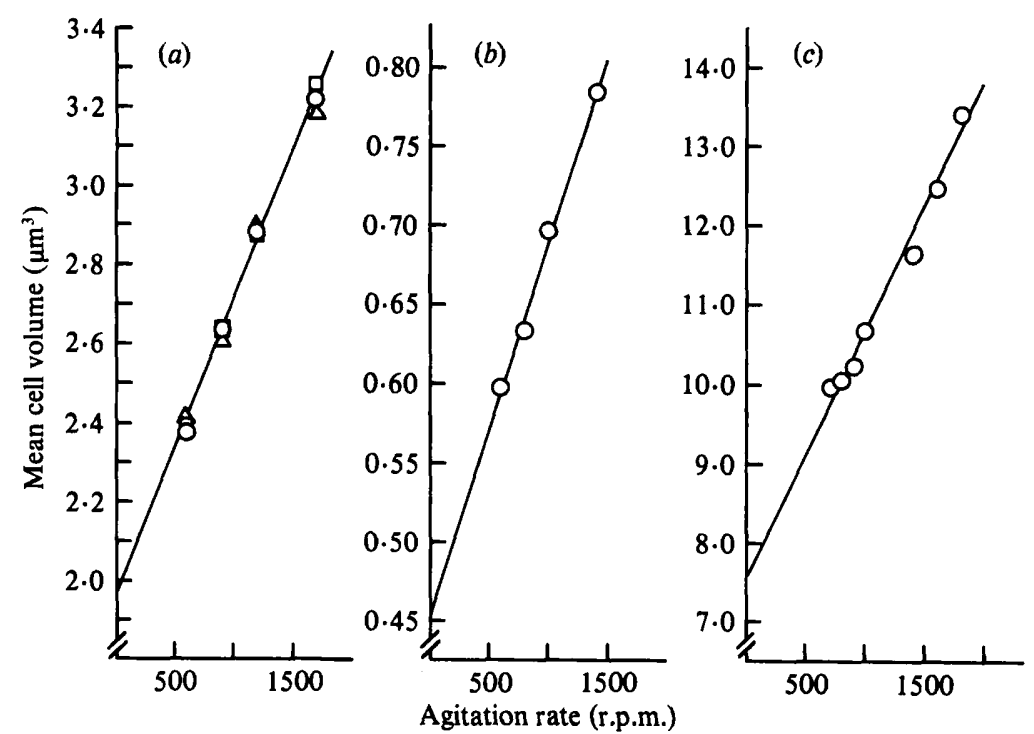

Fig. 3. Mean cell volumes of continuously cultivated B. cereus, Staph. epidermidis and Sacch. cerevisiae at various agitation rates. All cultures were aerobic. See Table 1 for details. $(a)$ B. cereus; $\square, O, \triangle$, experiments 14, 15 and 16. (b) Staph. epidermidis; experiment 17. (c) Sacch. cerevisiae; experiment 18.

B. cereus, Staph. epidermidis and Sacch. cerevisiae: experiments 14-18) are summarized in Fig. 3. Once again, in each case, mean cell volume increased with increase in agitation rate, the correlations being very precisely linear; and again (for B. cereus), for any agitation rate, alteration in the overall strength of the medium altered the numbers of cells sustained by the system, but did not change the mean cell volume.

\section{Total cell counts, viable cell counts and dry weights}

Because continuous systems were used, medium was supplied at a constant rate, and it would be reasonable to expect increases in mean cell volume of the magnitude we have observed (up to $50 \%$ volume increase) to be countered by changes in viable or total cell counts or in dry weight estimations. However, measurements that were made did not provide such evidence. Typical data are provided for the two strains of $E$. coli in Tables 2 and 3, showing that the viable counts, 

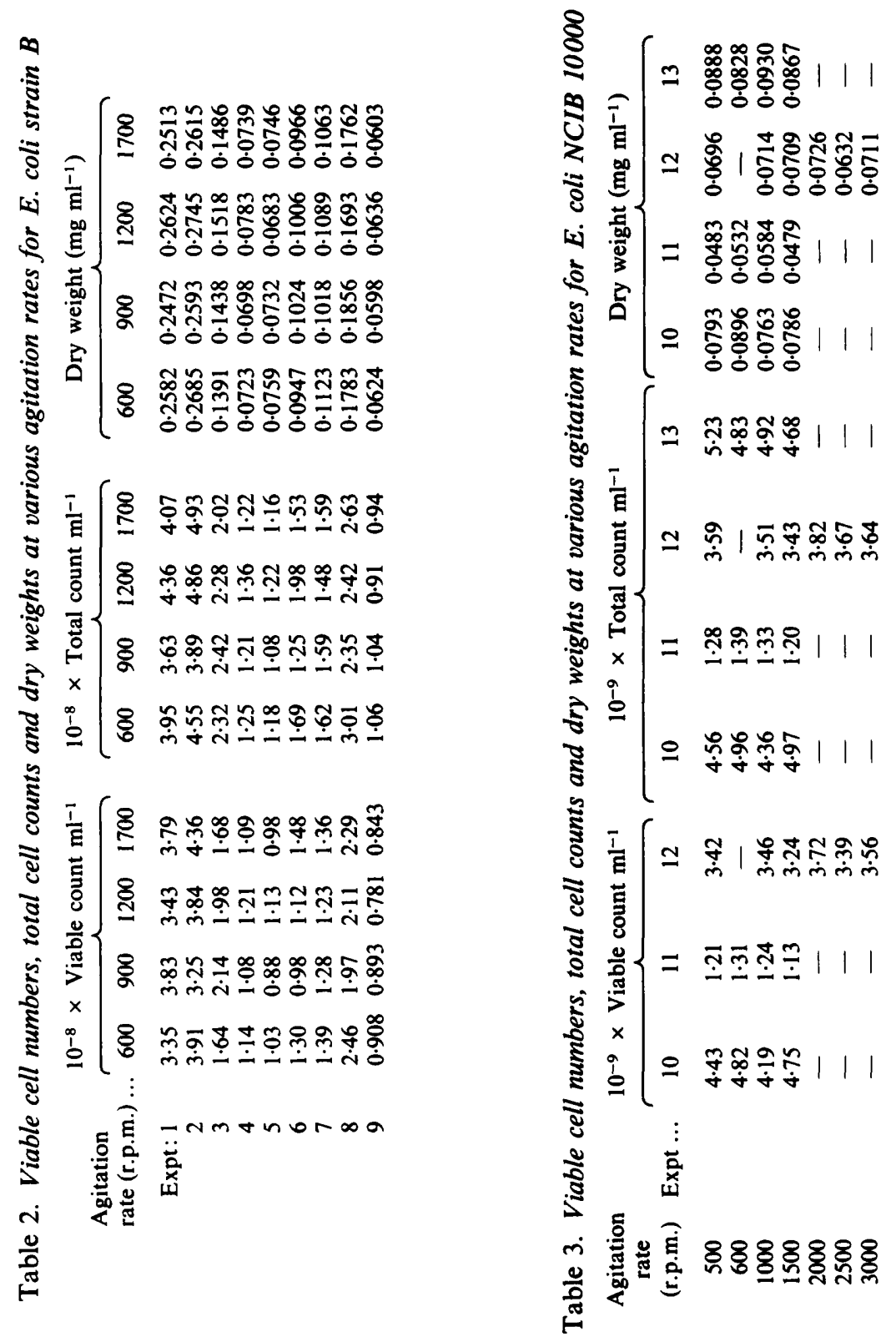

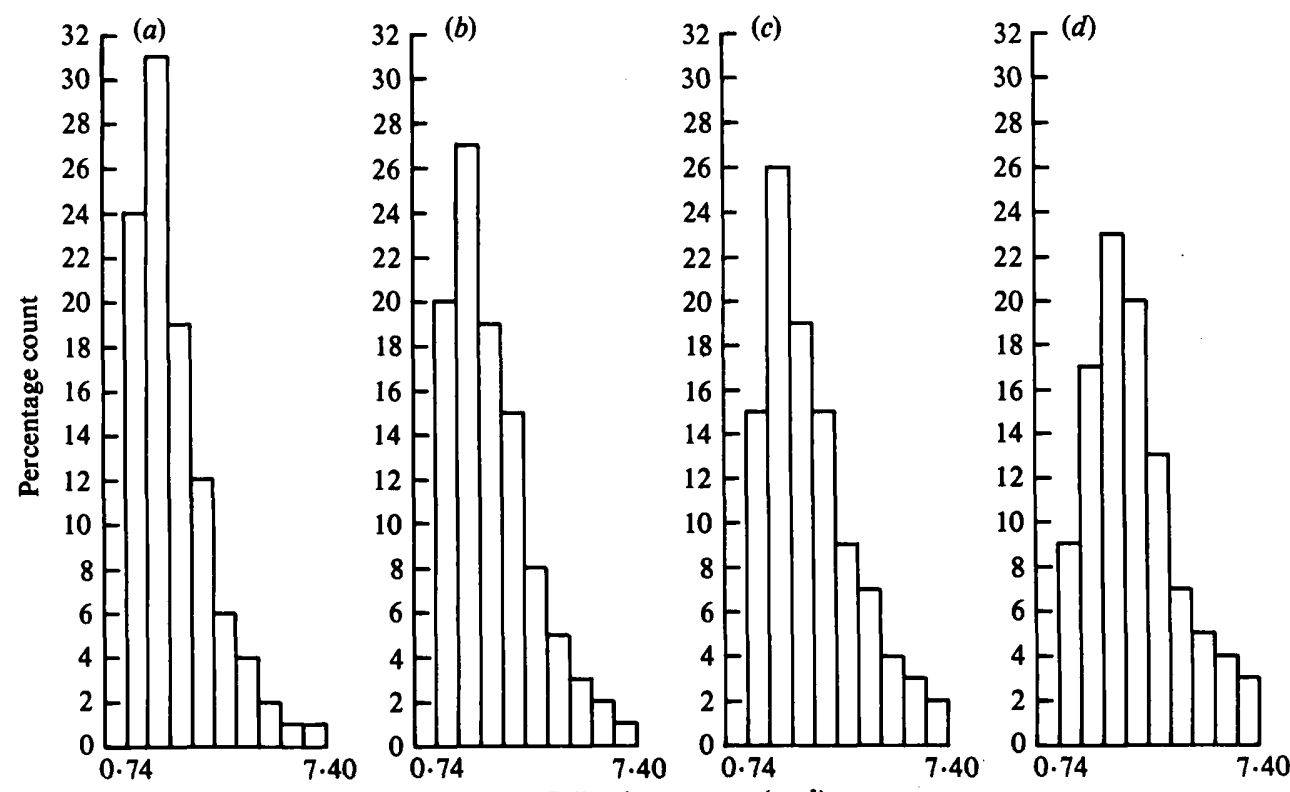

Fig. 4. Bacillus cereus, experiment 14. Histograms showing percentage distributions of cell volumes as measured by the Coulter Counter, at agitation rates of $(a) 600,(b) 900,(c) 1200$ and $(d) 1700$ r.p.m. The nine bars of each histogram represent, from left to right, the percentage of cells of volumes $0.74-1.48$, $1.48-2 \cdot 22,2 \cdot 22-2 \cdot 96,2.96-3 \cdot 70,3 \cdot 70-4.44,4.44-5.18,5.18-5.92,5.92-6.66$ and $6.66-7.40 \mu \mathrm{m}^{3}$ respectively; the highest bar, $7 \cdot 40-8 \cdot 14$, was not measured as this is subject to considerable error.

total counts and dry weights were essentially constant for each strain tested, although there was considerable scatter. Similar data were obtained for B. cereus, Staph. epidermidis and Sacch. cerevisiae (Tables 4 and 5).

In experiments 12 and 13 in the 'Baby' fermenter (Table 3) there were differences in the dry weights measured between the two experiments: the figures obtained with the smaller impeller were significantly lower $(27 \%)$ than those with the larger impeller. However, within each of the two experiments, the figures were sensibly constant, in spite of a change in mean cell volume of $24 \%$ with the larger impeller over the range of agitation rate studied. Therefore, the change in cell volume arising from a change in agitation rate seems, within any experimental run, to be almost wholly attributable to a change in the water content of the microbial cells, although the reasons for this are not clear.

\section{Cell size distribution}

Cells growing as films tend to revert from the 'dwarf' colonizers described by Marshall (1980) to normal or even large cells. To test the possibility that an increase in shear due to an increase in agitation rate removed some large cells from, say, an invisible layer on the fermenter walls, histograms were constructed of the data from the Coulter Counter; a typical example is shown in Fig. 4. This shows that as the agitation rate was increased, so there was a smooth progression of cell size distributions with no obvious humps or discontinuities, and this possibility was therefore discounted. The Coulter Counter has been used for a number of studies on cell sizes and has been shown not to be influenced by microfibrils, pili or flagella (Lanfer et al., 1979; Parker \& Barnes, 1966).

It is, however, unwise to rely on one technique alone. Microscopic examination of unstained wet films was therefore undertaken. This confirmed that the sizes of the cells increased with increasing agitation rate.

An independent method of obtaining absolute indications of cell size is by photographing the cells. As previously mentioned, the change in the cell volume in any experimental run appeared 


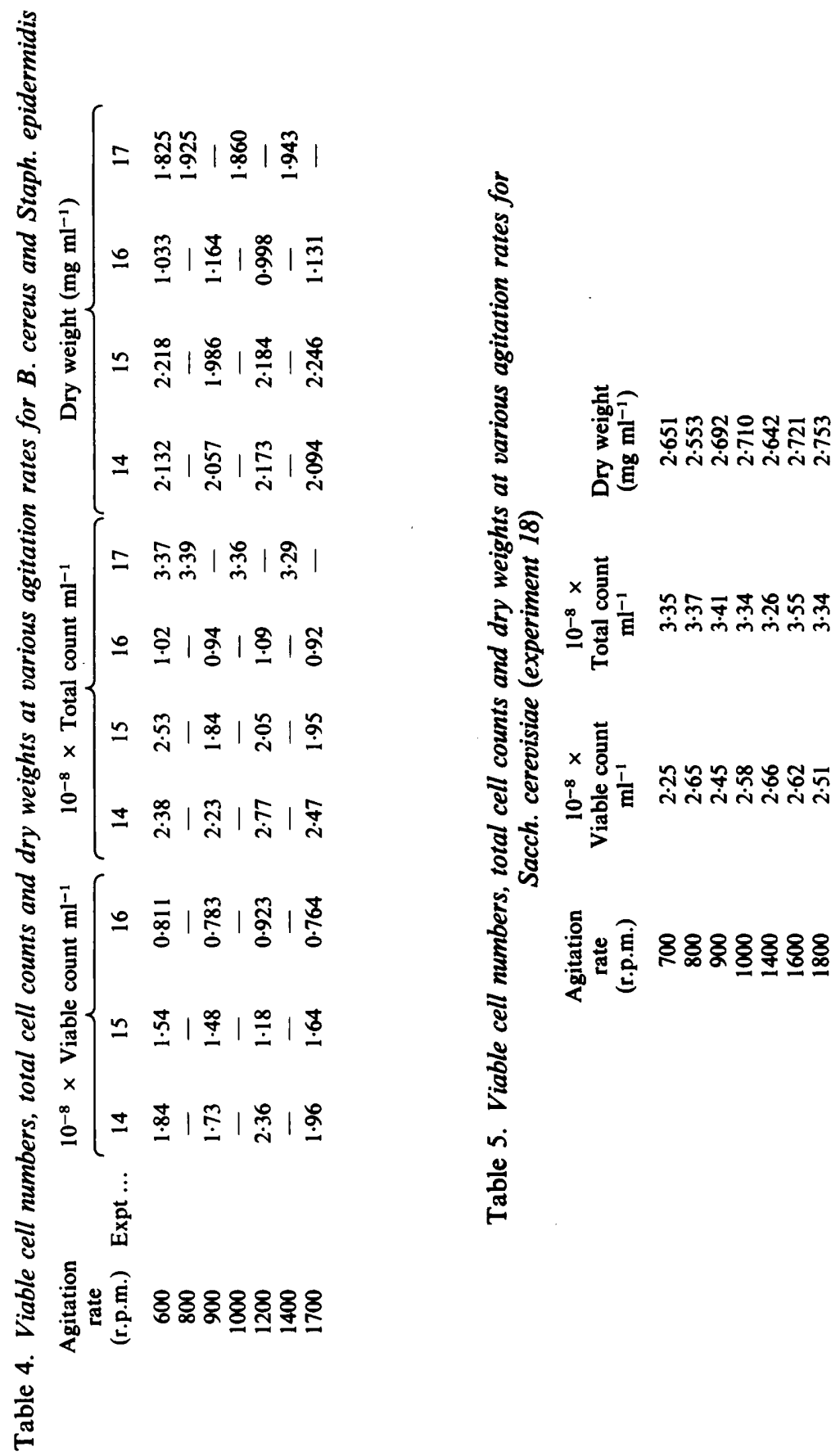



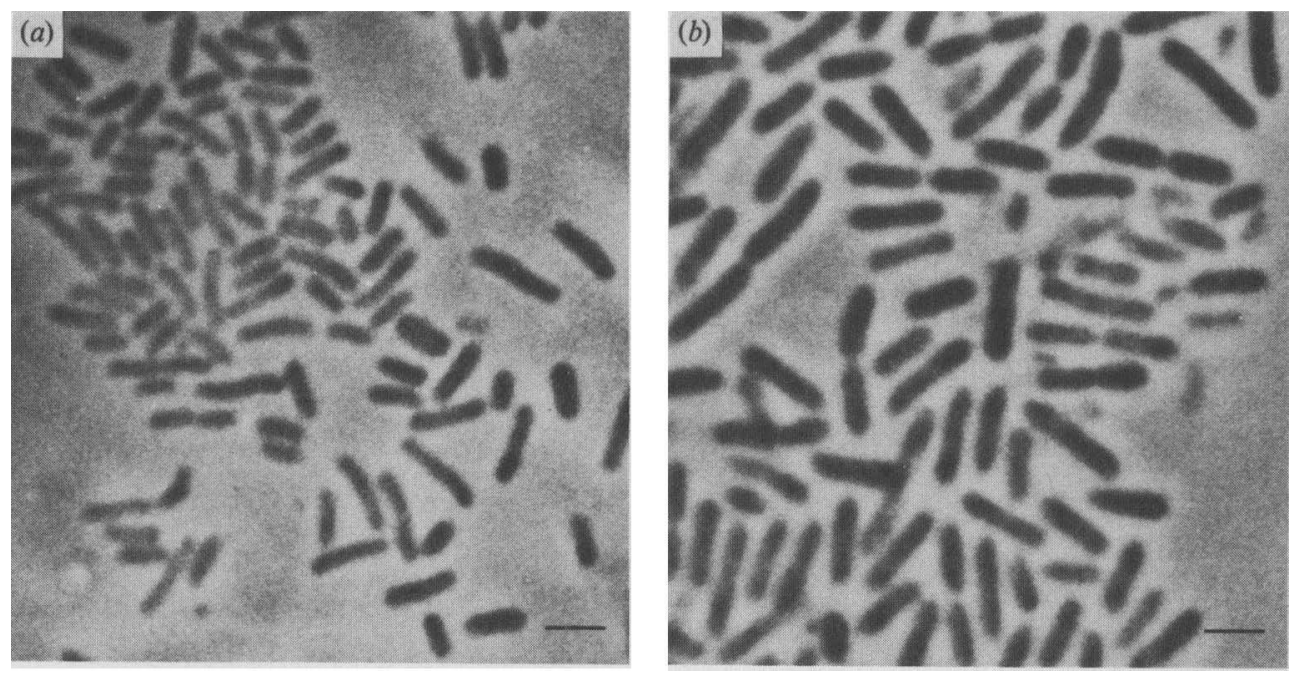

Fig. 5. Anaerobic carbon-limited continuously cultivated cells of $E$. coli NCIB 10000 grown in the 'Baby' fermenter equipped with the large impeller. (a) Small cells cultured at 500 r.p.m. (b) Large cells cultured at 1500 r.p.m. The bar markers represent $2 \mu \mathrm{m}$.

to be attributable to a change in water content, and if this is so it is then not advisable to use fixed stained films, because this procedure is likely to cause migration of intracellular fluid out of the cells: those with the highest water content could then lose most water. Instead, the alternative technique of mounting an unstained preparation in petroleum jelly (see Methods) was used: whilst this led to some loss of definition, the resulting photographs (Fig. $5 a, b$ ) demonstrate unequivocally and independently the changes that were recorded by the Coulter Counter.

\section{Agitation-rate-induced damage}

A further effect of agitation rate increase is the possibility of damage to the cells by shear. However, comparison of Figs $5(a)$ and $5(b)$ does not suggest any such damage. In addition, release of protein would almost certainly have caused foaming problems of the type limiting the extent of the Staph. epidermidis culture on complex medium, and these were not evident. Larger cells have been shown to be more easily disrupted (Thacker, 1973; Nesaratnam et al., 1982). It is hard to see how shear damage could lead to a stable population of larger cells. It was concluded that shear-induced damage was unlikely to produce the observed effects.

\section{Oxygen transfer effects}

Fig. $1(a, c)$ shows that in aerobic cultures, dissolved oxygen could not be maintained at $100 \%$ saturation throughout the range of agitation rates used. Variation in dissolved oxygen was ruled out as the cause of the effects, since $(a)$ the curves relating dissolved oxygen content and agitation rate are non-linear, $(b)$ over the regions where approximately $100 \%$ saturation is achieved the corresponding relationship between mean cell volume and agitation rate still remains precisely linear, $(c)$ exactly similar correlations hold when the medium concentration (and hence the oxygen demand) is halved, and $(d)$ similar effects were obtained in anaerobic culture when oxygen was absent.

\section{Other mass-transfer considerations}

In general terms, an increase in agitation rate in a disc turbine impeller system will increase both pumping action and shear forces. This, in turn, could lead to improvements in distribution of nutrient molecules around the microbial cell and a potential reduction in the thickness of the static film around the micro-organism with a consequent potential improvement in nutrient mass-transfer. 
In any continuous cultivation system at equilibrium, a change in the concentration of limiting nutrient leads to a corresponding change in the numbers of cells (Herbert et al., 1956); indeed this is shown to be so in the present case in the results for full-strength and half-strength medium (Tables 2 and 4). It can be argued that improved availability of limiting nutrient due to masstransfer improvements would be likely to have a similar effect, and so cell numbers would be likely to rise with increase in agitation rate. However, this is not so. Improvements in the supply of non-limiting nutrient are more likely to lead to cells which store materials such as carbohydrate and hence to dry weight increases with increase in agitation rate. Neither of these suggestions explains our observations of sensibly constant dry weight and cell numbers with an increase in size (which appears to be due almost wholly to increased water content), with increase in agitation rate. Indeed this point is particularly clearly shown by comparisons between the two sets of data obtained with the large and small impellers in the 'Baby' fermenter (small impeller, experiment 12; large impeller, experiment 13). Over the range of impeller speeds tested, the smaller impeller has far less capacity for mixing and for mass-transfer than the larger impeller, and this is reflected in the figures for cell numbers and dry weights (Table 3 ). However, it is important to note that within both of the two experimental runs, these figures remained sensibly constant, although in absolute terms they were smaller for the smaller impeller. In contrast, the mean cell volume varied within each experimental run, increasing linearly with agitation rate. This phenomenon is therefore quite distinct.

\section{Possible errors in continuous cultivation}

Laboratory continuous cultivation systems are subject to a number of problems leading to errors. One problem already mentioned arises from the formation of films of micro-organisms, sometimes evident in the vessel headspace, and care was taken to avoid such systems. Another source of difficulty is uneven pumping: uneven addition of medium has been shown by Dawson (1972) to result in effects leading to partial synchrony of cultures. The pumps used in this study were all peristaltic, and chosen because of their uniform flow characteristics, to avoid this problem as far as possible.

The laboratory air-supply is unlikely to be saturated with water vapour. However, for fermenters of the capacity and shape that we used, the effluent air stream is almost always completely saturated. This can cause medium evaporation errors which could vary with agitation rate, leading, in turn, to apparent changes in the nutrient concentration. However, this source of error is likely to be constant over the range of agitation rates employed in aerobic culture, and is eliminated in the anaerobic systems.

A further problem is the measurement and control of working volume. This is affected by the liquid level when weir devices are employed (increase in agitation rate causes increased surface turbulence which results in a smaller fluid volume). This difficulty can be obviated by a pumped weir. Increased agitation rate also causes increases in gas hold up at constant fluid (gassed liquid) volume : this again results in a progressive decrease in the liquid volume. For the 'Bioflo' and 1.3 litre fermenters, these two potential sources of error were compensated for by careful adjustment of the pumping rate, as mentioned earlier.

However, this compensation gives rise to a further error : the ratio of impeller size to working volume effectively increases with decrease in working volume. Although small, this change cannot be compensated for. Fortunately, none of the last three difficulties arises when working volume is kept constant by means of a load cell, and this type of fermenter could therefore be used to confirm our observations.

\section{Concluding remarks}

The effect reported in this paper has a number of possible applications. For instance, preliminary results have already been published showing that for ultrasonic disintegration a linear relationship exists between the disintegration constant and the mean cell volume (Nesaratnam et al., 1982) and further work in progress confirms that this observation applies to a wide range of organisms. It thus appears possible to arrange for an organism to be only just strong enough to withstand the rigours of transfer from fermenter to disintegration equipment, 
with a consequent saving in power costs in the disintegration stage. Indeed, since the change in mean cell volume is rapid, and virtually complete after three fermenter volume changes, there exists the possibility of incorporating equipment to effect such a change in the downstream processing regime of a continuous system. In a completely different application of our effect, we are currently seeking correlations between mean cell volume and the transformation rate, aimed at producing cells in which transformation rate matches the experimenter's requirements.

Finally, in summary, it is concluded that the effect we have observed appears to be very general, being applicable to a wide range of micro-organisms and cultivation conditions. Since its extent has been shown to differ both from one impeller to another, and also for different vessels, it is likely to be intimately associated with the geometry and action of the agitation system, but in a manner which we do not at present understand. Currently, the experimental programme has been concentrated on measurements of intracellular solute concentrations for cells of differing sizes which we hope will shed some light on the mechanisms governing this interesting phenomenon.

We are grateful for financial support for this work from the Science and Engineering Research Council.

\section{REFERENCES}

Brown, C. M. (1966). Metabolic studies of yeast in continuous culture. $\mathrm{PhD}$ thesis, University of Birmingham, UK.

Brown, C. M. \& Hough, J. S. (1965). Elongation of yeast cells in continuous culture. Nature, London 206, 676-678.

Dawson, P. S. S. (1972). Continuously synchronised growth. Journal of Applied Chemistry and Biotechno$\log y$ 22, 70-103.

Dean, A. C. R. \& Hinshelwood, C. (1966). Growth, Function and Regulation in Bacterial Cells, pp. 87-89. Oxford: Clarendon Press.

Herbert, D. (1961). The chemical composition of microorganisms as a function of their environment. Symposia of the Society for General Microbiology 11, 391-416.

Herbert, D., Elsworth, R. \& Telling, R. C. (1956). The continuous culture of bacteria: a theoretical and experimental study. Journal of General Microbiology 14, 601-622.

KJelleberg, S., Humphrey, B. A. \& Marshall, K. C. (1983). Initial phases of starvation and activity of bacteria at surfaces. Applied and Environmental Microbiology 46, 978-984.

Lanfer, N., MaY, J. Y., Segar, S. \& Grover, N. B. (1979). Size of rat spermatozoa during maturation along the epididymis. Archives of Andrology 3, 293299.

MarshaLL, K. C. (1980). Bacterial adhesion in natural environments. In Microbial Adhesion to Surfaces, pp.
187-196. Edited by R. C. W. Berkeley, J. M. Lynch, J. Melling, P. R. Rutter \& B. Vincent. Chichester: Ellis Horwood.

Nesaratnam, S. T., Wase, D. A. J. \& Blakebrough, N. (1982). The susceptibility to ultrasonic disintegration of Klebsiella pneumoniae NCTC 418. European Journal of Applied Microbiology and Biotechnology 15, 56-58.

PARker, M. S. \& BARnes, M. (1966). Use of the Coulter Counter to measure the swelling of bacterial spores during germination and outgrowth. Journal of Applied Bacteriology 30, 299-303.

PIRT, S. J. (1975). Principles of Microbe and Cell Cultivation, chapters 3 and 15. Oxford: Blackwell Scientific Publications.

THACKER, J. (1973). An approach to the mechanism of killing of cells in suspension by ultrasound. Biochimica et biophysica acta 304, 240-248.

Wase, D. A. J., Nesaratnam, S. T. \& Blakebrough, N. (1982). Variation in cell-wall strength of Klebsiella pneumoniae NCTC 418 with change in agitation speed in a chemostat system. Journal of Chemical Technology and Biotechnology 32, 553-555.

WICKERHAM, L. J. (1951). US Department of Agriculture Technical Bulletin no. 1029.

WIMPENNY, J. W. T. \& FiRTH, A. (1972). Levels of nicotinamide adenine dinucleotide and reduced nicotinamide adenine dinucleotide in facultative bacteria and the effect of oxygen. Journal of Bacteriology 111, 24-32. 